\title{
LA REHABILITACIÓN Y RESTAURACIÓN DE MONUMENTOS Y CIUDADES HISTÓRICOS EN CHECOSLOVAQUIA
}

\section{(THE RENOVATION AND RESTORATION OF HISTORIC MONUMENTS AND CITIES IN CZECHOSLOVAKIA)}

\author{
Fernando Aguirre de Yraola, Dr. Arquitecto \\ ICCET/CSIC/ESPAÑA
}

\section{RESUMEN}

Contiene el presente articulo una sintesis de las ponencias que se presentaron al Simposio sobre "Rehabilitación de centros y monumentos históricos" que se han celebrado recientemente en Praga, organizado por la asociación técnico-científica europea EUROBUILD.

Además de las citadas sintesis, se da una información referente a unas interesantes visitas técnicas realizadas por los participantes al Simposio, en especial las redes subterráneas de los servicios para una óptima rehabilitación de los núcleos históricos de Praga y Tábor, asi como de los problemas planteados y los métodos geotécnicos empleados.

\section{SUMMARY}

This article contains a summary of the papers presented at the Symposium on "Renovation of historic centres and monuments" which was held recently in Prague and organised by the European technical-scientific association EUROBUILD.

In addition to the aforementioned summaries, information is given on some interesting technical visits made by those attending the Symposium, particularly the underground service networks for optimum renovation of the historical centres of Prague and Tabor. The problems that occurred and the geo-technical methods used are also described.
Organizado por EUROBUILD, la sociedad europea que desde hace más de veinte años viene convocando Sim. posios y Conferencias sobre los temas que más interesan en cada momento en el campo de la construcción, y cuyo organismo directivo está compuesto por los más prestigiosos centros científico-técnicos y empresariales de dicho campo, ha tenido lugar recientemente en Praga un Simposio sobre "Rehabilitación y Restauración de Monumentos y Ciudades Históricos".

Participaron en este Simposio numerosos arquitectos, urbanistas, ingenieros civiles, químicos y mecánicos, especialistas de la información y representantes de empresas constructoras y de organización, los cuales expusieron ponencias especializadas que sirvieron para posterior coloquio. También se realizaron interesantes visitas técnicas dentro de la ciudad de Praga y en otras ciudades y monumentos checos.

Checoslovaquia pertenece, en realidad, al grupo de naciones que poseen una alta densidad de monumentos culturales, los cuales han recibido gran protección le-

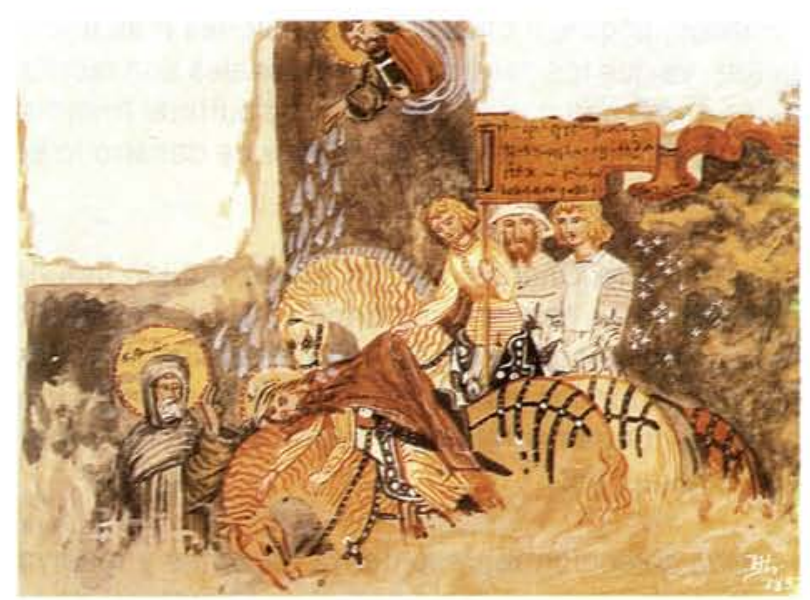

gal por parte del gobierno. En 1978, UNIDO y el gobierno de la República Socialista checa, acordaron establecer un Programa conjunto de cooperación internacional. Uno de los objetivos de este programa ha sido el de los trabajos de restauración y rehabilitación de 


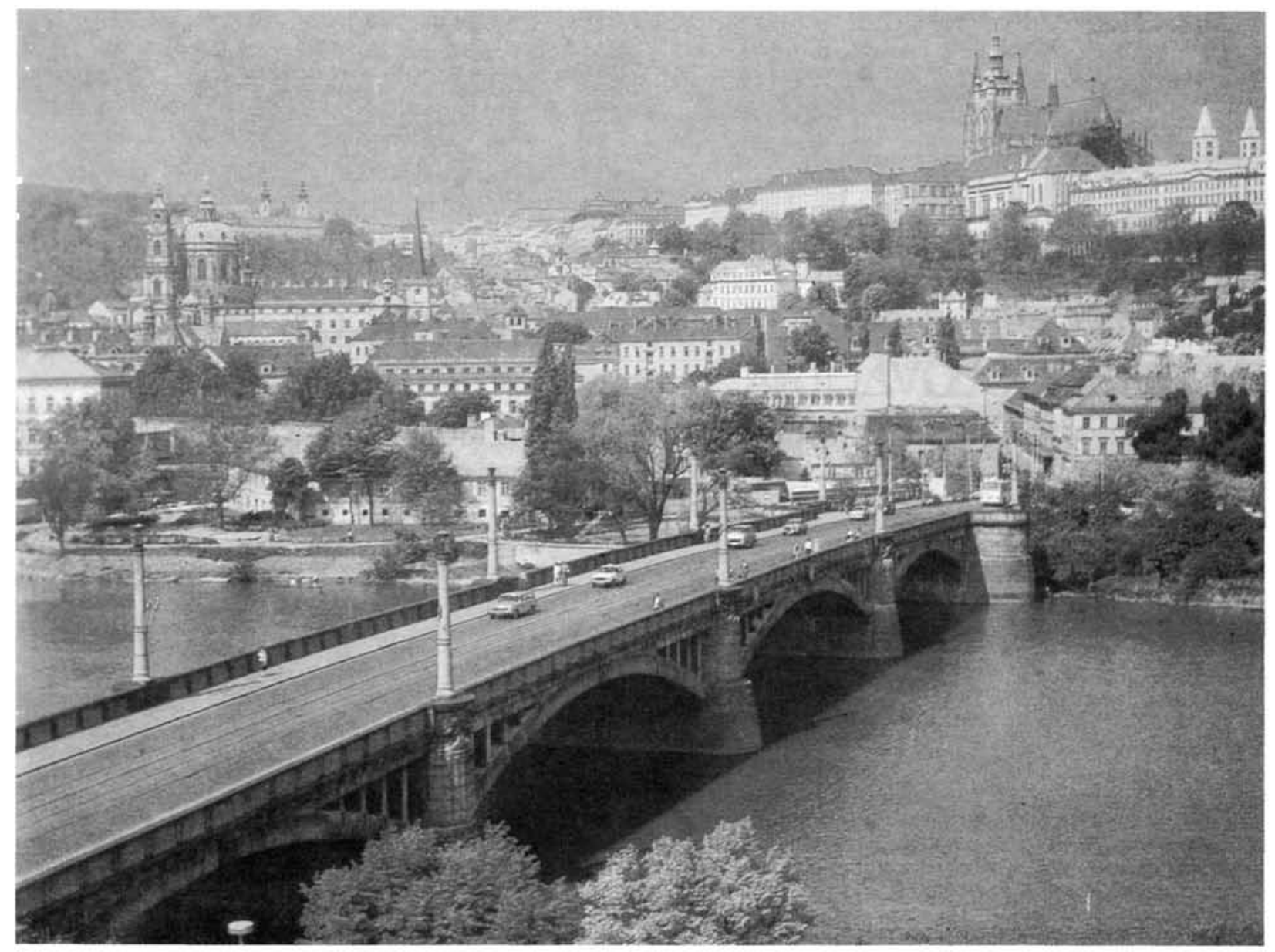

Fig. 1.-Vista de Praga, desde el rio VItava.

monumentos y centros históricos, y este hecho supone una manifestación de que la protección de los edificios históricos, común para todos los países desarrollados, adquiere cada vez dimensiones más importantes, ya que los patrimonios nacionales son reconocidos como una parte de la herencia cultural mundial, aunque reflejen unas características de desarrollo especificas para cada pais.

Por otra parte, Checoslovaquia posee, debido indudablemente a su herencia histórica, una de las metodologias para la conservación y la protección de monumentos históricos más elaboradas y desarrolladas, que consiste fundamentalmente, según el ponente Frantis̃ek Soukup, en:

- Una selección representativa de zonas y reservas históricas, de acuerdo con el valor cultural de las necesidades de la sociedad checa.

- Una actuación diferenciada sobre la protección de los monumentos históricos mediante un régimen estricto en las reservas homogéneas, y algo más flexible en las zonas con una administración diferenciada.
Se ha comprobado que el crecimiento unilateral del área y de la población de centros históricos por medio de nuevos esquemas de viviendas construidas en sus partes marginales, a veces lejos de satisfacer todas las necesidades sociales, se ve limitada por serios factores e impone exigencias crecientes en el servicio, distribución y funciones sociales de las zonas centrales y de los sistemas de comunicación.

Las partes centrales de los conjuntos históricos necesitan regenerarse completa e inevitablemente, para que puedan continuar desempeñando sus importantes funciones y para emprender nuevas actividades, por lo cual necesitan ser renovadas, reconstruidas y modernizadas.

El sistema de asentamiento histórico, asi como los esquemas y zonas individuales, no alcanzarán probablemente una situación final, pero formarán un sistema abierto que se desarrollará de acuerdo a cómo puedan controlarse sus factores de aceleración o deceleración.

Por lo tanto, las ciudades y conjuntos históricos no pueden desarrollarse de modo unilateral mediante una reconstrucción in extenso, sino mediante el incremento 
de la intensidad de su utilización funcional y la valoración de sus calidades arquitectónicas en el marco del proceso paralelo de una regeneración continua e integral.

Por otra parte, el problema de la reconstrucción de edificios históricos adquiere un alto grado de complejidad si el edificio resulta dañado tan seriamente que amenace una inminente destrucción, o incluso que ya haya quedado destruido. En estos casos, los trabajos de reconstrucción deben ir precedidos de medidas preventivas seleccionadas cuidadosamente y no han de interferir con los subsiguientes conceptos de renovación, y uno de los requisitos para que esto se realice eficazmente es el diagnóstico de láminas y grietas, mediante adecuados métodos estáticos, geológicos, etcétera.

Según el ponente Jan Svejcha, pueden distinguirse tres grados básicos de deterioro en la fábrica de ladrillo de un edificio histórico:

1) La fábrica de ladrillo se halla preservada, total o en su mayor parte.

2) La fábrica de ladrillo ha sido muy demolida, pero los escombros dan idea de la disposición original.

3) Sólo quedan montones de cascotes.

En la mayoria de los casos se mantiene y preserva el carácter pragmático del edificio histórico, se salva lo que ha quedado de la fábrica de ladrillo, y se lleva a cabo el suplemento más esencial de las partes resistentes, principalmente para estabilizarlas. Se procede meticulosamente de manera que las partes nuevas de la fábrica sean sólo una reconstrucción de las formas originales y no constituyan nuevas formas indeseables. Se emplea material de cascote para suplementar y se adapta la técnica de albañileria a la fábrica de ladrillo existente. Todo esto se aplica también a las bóvedas reconstruidas total o parcialmente.

En casos excepcionales y principalmente tratándose de edificios de especial importancia, se emplea la llamada reconstitución científica, es decir, se reconstruye la ruina en su forma original, de la cual existe información auténtica y fiable o que se ha obtenido mediante estudios históricos estructurales.

Si solamente se dispone de una idea aproximada de su primitiva apariencia, se elige el método indirecto de reconstrucción, realizando el volumen y disposición del edificio original, y renovando y suplementando por medio de elementos hallados entre los cascotes de las ruinas, mientras que aquellas partes que no han podido ser preservadas se reconstruyen con nuevos materiales según nuevo diseño. Sin embargo, este diseño deberá armonizar con el carácter histórico del edificio.

Para la consolidación estática de las ruinas, en especial las que se hallan en estado avanzado, es necesa- ria una tecnología moderna, que requiere una mecanización potente, capaz incluso de actuar en sitios innaccesibles, y en terrenos afectados por procesos de descomposición; y la estabilización de edificios situados en formaciones rocosas exige estudios geotécnicos.

Un interesante ejemplo de resolución de estos problemas en Checoslovaquia lo constituye el Castillo de Orlik, situado en un acantilado escarpado y abrupto de 65 metros sobre el río Vltava y amenazado por el remanso de la presa de Orlik, cuya profundidad ahi es de 60 metros. La roca del acantilado se hallaba muy fisurada y dañada por numerosas fallas tectónicas. La albañilería del castillo, consecuentemente, también habia sufrido considerablemente.

En primer lugar, las cimentaciones del castillo fueron reforzadas hasta alcanzar la roca saneada, pero el principal problema era la conservación del empinado y casi vertical acantilado cara al Vltava.

Se construyó un chaleco de hormigón armado anclado al núcleo y reforzado por medio de un sistema de varillas pretensadas. Este chaleco resiste la superficie de la roca desde la marca correspondiente al mínimo nivel del agua del embalse, hasta la correspondiente al máximo nivel (Fig. 2).

La empresa nacional "Geoindustria" es la más importante, en Checoslovaquia, dedicada a trabajos de ex-

a) Orificios para, mediante repetidas compactaciones, reforzar la resistencia del subsuelo.

b) Varillas de anclaje.

c) Gunitado hidráulico para refuer. zo y anclaje.

d) Zona de falla.

e) Nivel inicial de Vitava (antes de la construcción de la presal.

f-g) Minimo-máximo nivel interno de la presa.

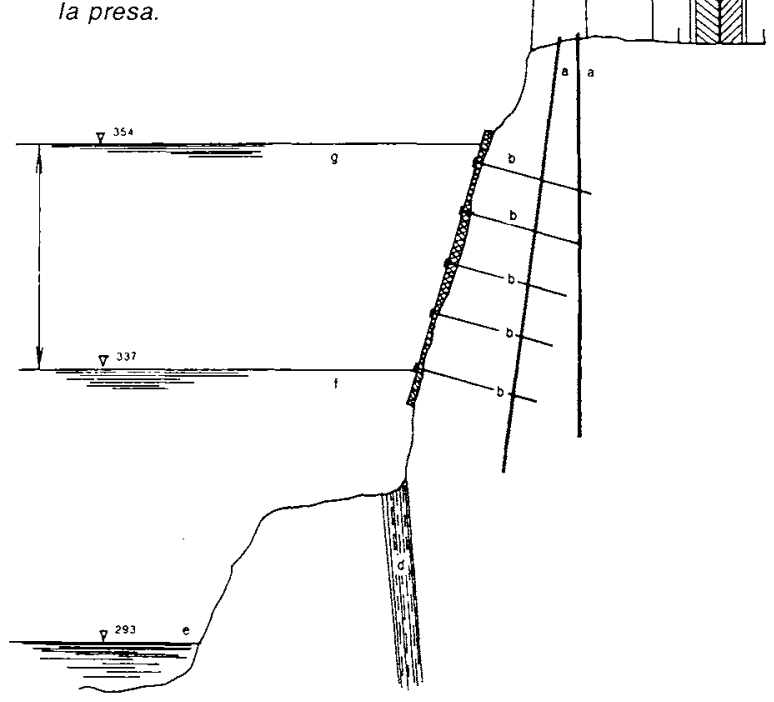

Fig. 2.-El castillo de Orlik, sobre el Vitava. 


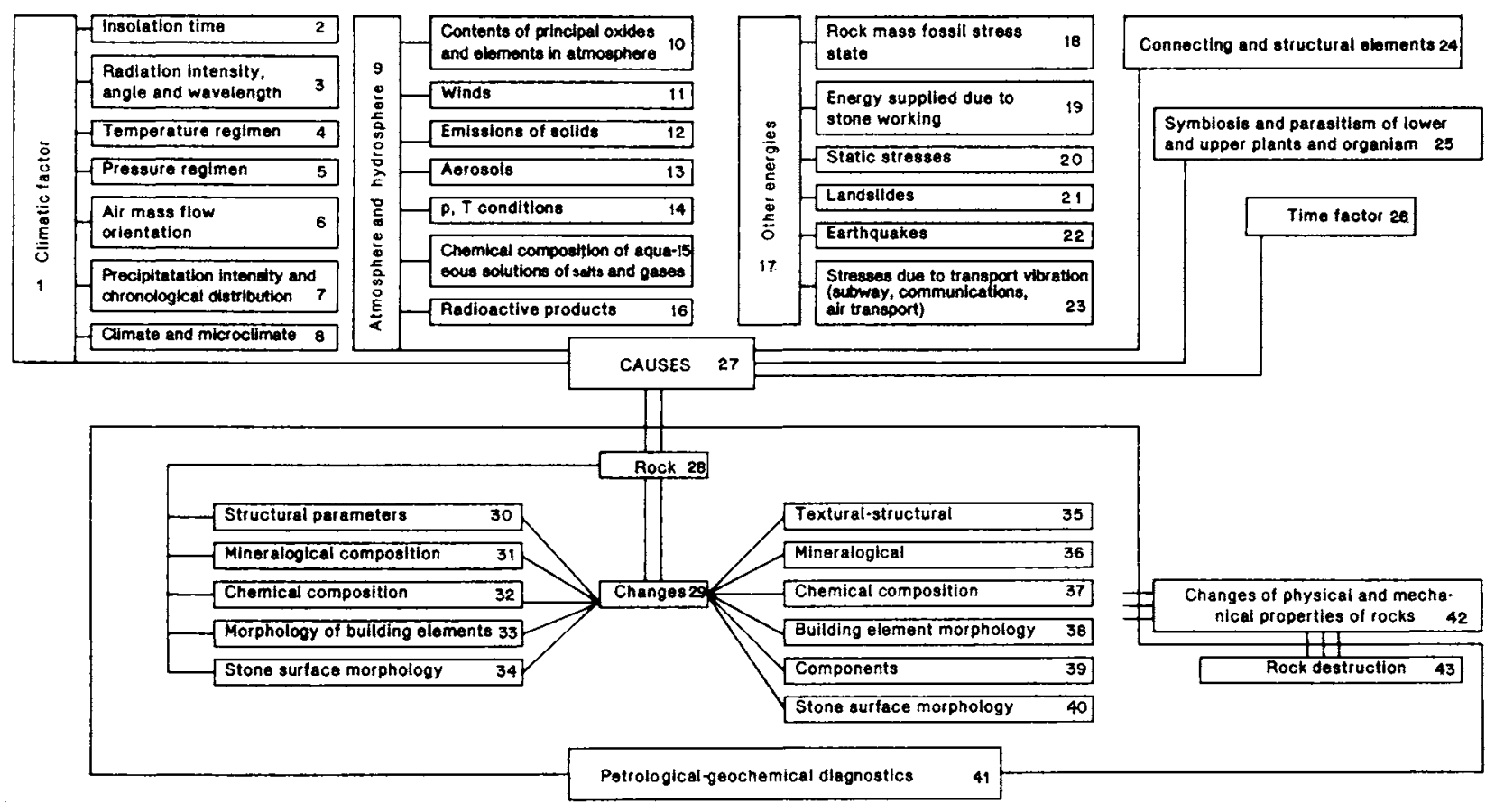

1.-Factor climático. 2.-Tiempo de insolación. 3.-Intensidad de radiación, ángulo y longitud de onda. 4.-Régimen de temperatura. 5.-Régimen de presión. 6.-Orientación del flujo de la masa de aire. 7.-Intensidad de precipitación y distribución cronológica. 8.-Clima y microclima. 9. - Atmósfera e hidrosfera. 10.-Contenido de los principales óxidos y elementos en la atmósfera. 11.-Vientos. 12. - Emisión de sólidos. 13.-Aerosoles. 14.-Condiciones de p. T. 15. - Composición química de soluciones acuosas de sales y gases. 16. - Productos radioactivos. 17. - Otras energias. 18. - Estado tensional de la masa de roca fósil. 19. - Energía suministrada al trabajo de la piedra. 20. - Tensiones estáticas. 21. Deslizamientos de tierras. 22. -Sismos. 23. Tensiones debidas a la vibración del transporte (metro, comunicaciones, transporte aéreo). 24.-Elementos estructurales y de conexión. 25.-Simbiosis y parasitismo de plantas inferiores y superiores, y organismos. 26. - Factor tiempo. 27.-Causas. 28. - Roca. 29. - Cambios. 30.-Parámetros estructurales. 31.-Composición nineralógica. 32.-Composición quimica. 33.-Morfologia de elementos de construcción. 34.-Morfologia superficial de la piedra. 35.-Textural-estructural. 36.-Mineralógica. ca. 33.-Morfologia de elementos de construcción. 34.-Morfologia superficial de la piedra. 35.-Textural-estructural. 36.-Mineralógica. 37.-Composición quimica. 38. - Morfología de los elementos de construcción. 39. Componentes. 40.-Morfologia superficial de la piedra. 41.-Diagnósticos petrológicos-geoquímicos. 42.--Cambios en las propiedades físicas y mecánicas de las rocas. 43. -Destrucción de la roca.

Fig. 3.-Diagrama esquemático de las causas de deterioro de las piedras utilizadas en la construcción y decoración de los edificios históricos.

ploración geológica. Esta empresa realiza modernos diagnósticos petrológicos y químicos como asesoramiento de proyectos de restauración y rehabilitación de edificios históricos.

Cada proyecto de este tipo se basa necesariamente no sólo en las condiciones estáticas y arquitectónicas observadas, sino también en el actual estado de deterioro de sus elementos estructurales. Los cambios sustanciales a lo largo del tiempo, debidos a factores externos, pueden dividirse en seis grupos básicos: factores climáticos; influencia de la atmósfera y la hidrosfera; influencia de otro tipo de energía; factores de tiempo; factores asociados con elementos estructurales de conexión; y simbiosis y parasitismo de plantas y animales, incluyendo el hombre.

Las influencias externas, cuya especificación viene dada en la figura 3, provocan una desintegración de las rocas, de los morteros y de los elementos decorativos en el tiempo y en el espacio, así como su descomposición, y un tipo especial de desgaste provocado por las condiciones atmosféricas, que recibe el nombre ge- nérico de "enfermedades de las piedras". Los modernos métodos de diagnóstico constituyen un prerrequisito esencial para sanar estas enfermedades, o para detener su avance.

El sistema de métodos de diagnóstico petrológico y geoquímico expuestos por el ponente Ludvik Cichovský puede aplicarse a cualquier tipo de rocas ígneas, metamórficas o sedimentarias empleadas en los edificios históricos checos. Se basa en la necesidad de descubrir, en forma cuantitativa, las cinco clases de parámetros enunciados anteriormente, es decir: morfología de la superficie de la piedra; caracteristicas estructurales; morfología de los componentes estructurales y de la porosidad; composición mineralógica; composición química de las piedras empleadas en construcción y decoración. Existen ciertos parámetros indicativos en cada clase, que pueden determinarse por métodos analíticos apropiados y después evaluarse sintéticamente, si es posible mediante ordenadores.

También existen modernos métodos de diagnóstico superficial basados en estudios microscópicos y análi- 

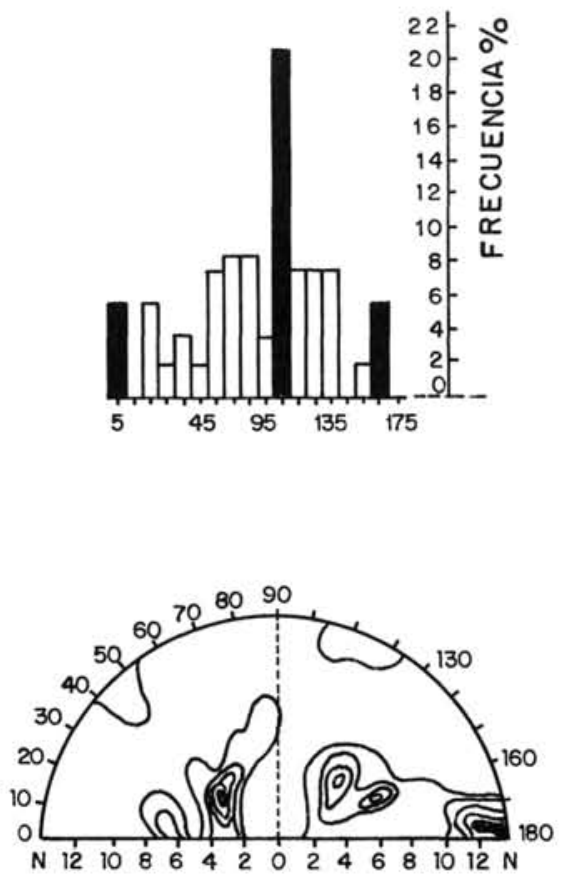

Fig. 4.-Desviaciones angulares.

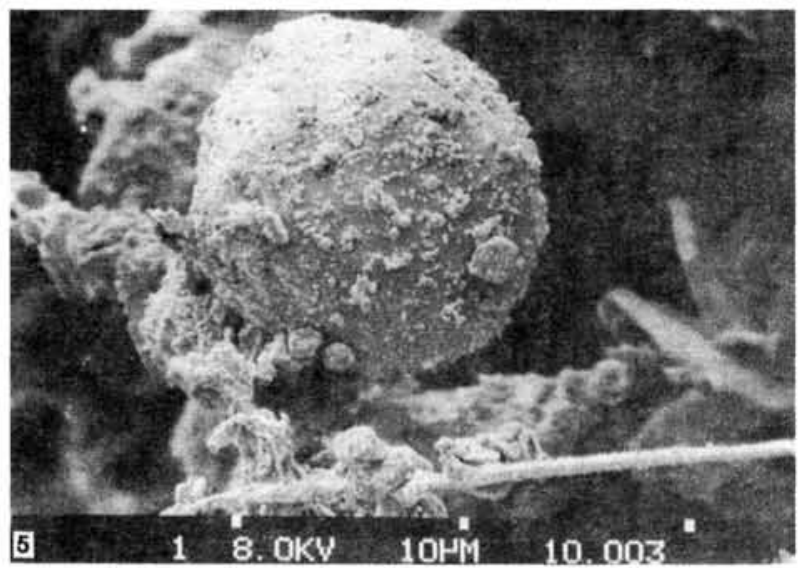

Fig. 5.-Vista detallada de la superficie de un grano de polvo adheri. do a la formación de costra en una arenisca silicea. (Puente de Carlos, Praga).

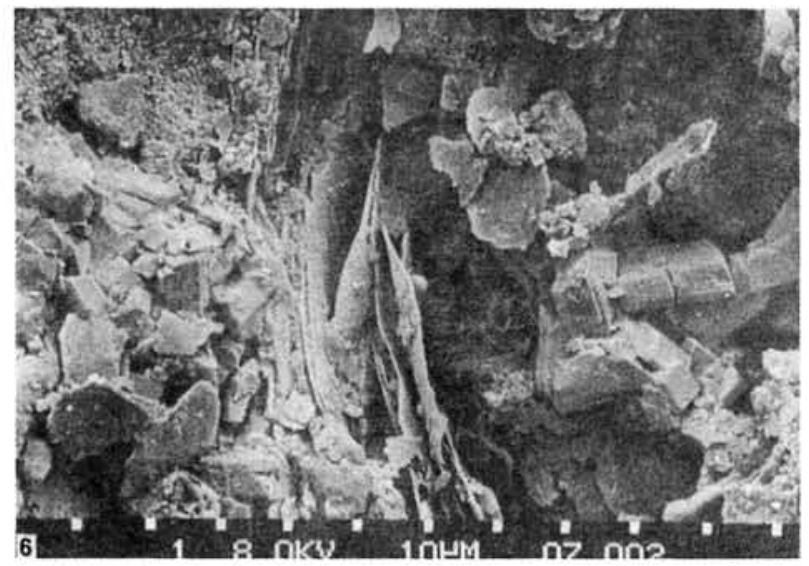

Fig. 6.-Fisura destructiva conteniendo un precipitado de yeso nue. vamente formado a partir de agregados de calcita disueltos. (c) Consejo Superior de Investigaciones Científicas Licencia Creative Commons 3.0 España (by-nc) sis mediante microcomputadores. Un ejemplo de esa evaluación lo tenemos en el Nuevo Ayuntamiento de Praga (Fig. 4).

Otro importante método consiste en una combinación del microscopio SCAN y del análisis EDAX, que se em. plea para determinar la morfometría y la composición quimica de las costras formadas. Éstas representan partes superficiales de las piedras, con un contenido dominante de minerales nuevamente formados, impurezas y organismos o plantas que existen simbiótica o parasitariamente en la superficie de la piedra (Ejemplos en las Figs. 5 y 6 ).

En resumen: el sistema de diagnóstico petrológico y geoquímico trabaja con parámetros esenciales para una apreciación cuantitativa del grado de deterioro (profundidad, tipo de daño, tipo de reacciones y cambios en la roca, etc.) asi como de los cambios estructurales, morfológicos, mineralógicos y químicos de las piedras empleadas en construcción y decoración en un medio agresivo.

Los materiales de soporte muestran solamente una fracción de la información cuantitativa que ha de determinarse de una manera objetiva antes de que un experto pueda decidir cómo proceder en la reconstrucción y la preservación de las piedras empleadas en construcción y decoración en un edificio histórico determinado.

La restauración de los centros de las ciudades, es decir, de las partes antiguas e históricas, implican la renovación de las fachadas de los edificios, la vuelta a las funciones originarias de las plantas bajas o de los soportales de aquellos (tiendas, restaurantes, solares de exposición, etc.) y el traslado de oficinas y organismos que no deben pertenecer a la vecindad, así como la extensión de las superficies para viviendas.

Todo esto representa un gran trabajo de preparación y realización y todos los cambios necesarios crean los graves problemas referentes a las normas de equipamiento - suministros de agua, calefacción, energia, etcétera-. Es necesario prever las influencias de las construcciones individuales, de sus trabajos de conservación, rehabilitación, mantenimiento y reemplazamiento respecto del entorno en que se hallen los edificios.

Las visitas técnicas que se realizaron durante el Simposio a los colectores al centro de Praga y de la ciudad de Tábor fueron enormemente ilustrativas de cómo pueden resolverse los grandes problemas que surgen en las ciudades históricas, cuando se procede a la rehabilitación de sus zonas monumentales y se desea evitar el deterioro que supone la introducción en ellos de los servicios y tecnología modernos.

http://informesdelaconstruccion.revistas.csic.es 
Las actuales condiciones en Praga se caracterizan por una actual falta de capacidad y una obsolencia en la distribución de agua. Las pérdidas de este elemento a través de las tuberias representan una tercera parte del caudal del suministro. Los cables distribuidores de la energía eléctrica necesitan ser reforzados y repuestos en varias zonas. Las débiles distribuciones de corriente no hacen posible satisfacer ni los requisitos de las instalaciones telefónicas, ni un incremento futuro necesario para las comunicaciones. También es necesario reforzar las distribuciones de gas y aumentar su seguridad. Las distribuciones de calefacción a partir de centrales no existen, y dado que el número de apartamentos calefactados localmente, sobre todo con fueles sólidos es alto, todo ello hace que las condiciones citadas afecten desfavorablemente al medio ambiente.

Existen varios medios para resolver esta situación. Hasta ahora, los intentos para reemplazar o reparar los elementos dañados eran principalmente a base de excavaciones, y la introducción de nuevas o restañadas cañerías en zanjas. Sin embargo, el número de excavaciones es enormemente alto, y no hay suficiente espacio para introducir las cañerias, sobre todo las principales o maestras, en las angostas calles.

Praga realiza considerables esfuerzos para constituir un sistema de canales subterráneos, galerias y túneles, en los cuales se pueden colocar los conductos principales y las cañerías secundarias para ser controlados, reparados o repuestos en aquéllos.

La sociedad estatal Interprojekt Praha preparó en 1981 un proyecto y en 1984 el Comité nacional de la ciudad de Praga autorizó la constitución de dos sistemas conectados entre sí, según el "Plan general de colectorización de la zona central de Praga”.

Según el ponente del tema, Ingeniero Frantis̃ek Dvorák, se han dispuesto grandes tuberías de 400-800 mm de diámetro para agua; de $500 \mathrm{~mm}$ de diámetro para gas; hasta 800 piezas de cables para comunicaciones, cables de electricidad para voltajes de $110 \mathrm{kV}$ y $22 \mathrm{kV}$, todos ellos introducidos en canalizaciones a una profundidad media de 22 metros.

Los conductos principales de distribución van colocados dentro de colectores próximos a la superficie, y distribuyen las tuberias secundarias hasta las entradas de servicio. Estos colectores se hallan conectados con los colocados a gran profundidad por medio de columnas de salida, y están en contacto con los edificios en la superficie, debajo de calzadas y aceras, y excepcionalmente debajo de los edificios o en el interior de éstos.

La separación de túneles y galerias entre los conductos principales y los sistemas de distribución es inevitable, ya que el complejo de los conductos principa(c) Consejo Superior de Investigaciones Científicas Licencia Creative Commons 3.0 España (by-nc) les requiere la construcción de túneles de tales dimensiones que no pueden colocarse en capas superficiales por las condiciones geológicas o hidrológicas y la existencia de otras estructuras subterráneas.

Para el abastecimiento del centro de Praga (con una superficie de unas $1.400 \mathrm{Ha}$ ), se ha propuesto una red de colectores profundos de $35 \mathrm{~km}$ de longitud, desde los cuales se conectan los colectores superficiales (con una longitud de $250 \mathrm{~km}$ ) por medio de 250 columnas (Figs. 7, 8, 9 y 10).

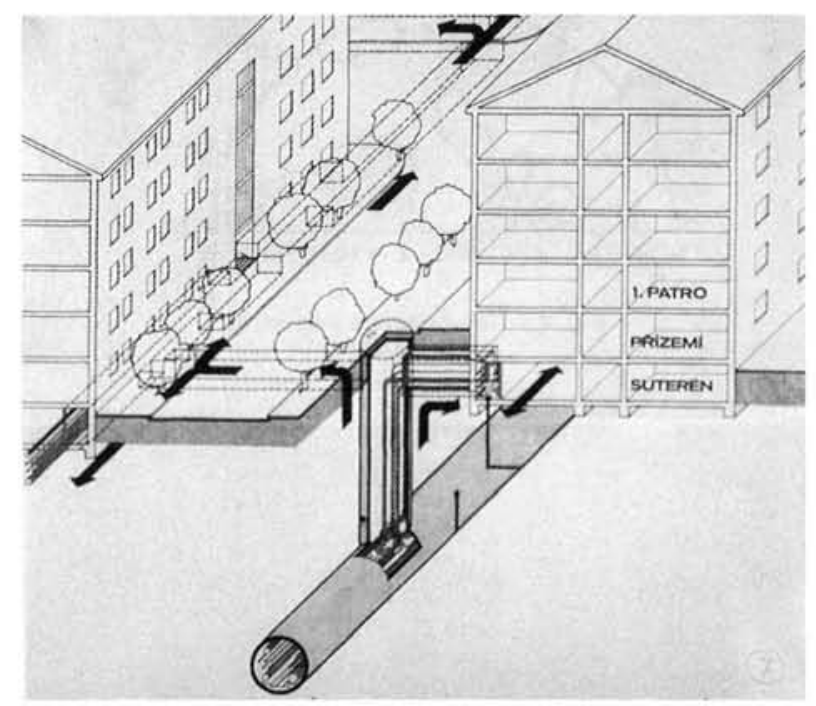

Fig. 7.-Sección del sistema de colectores de Praga.

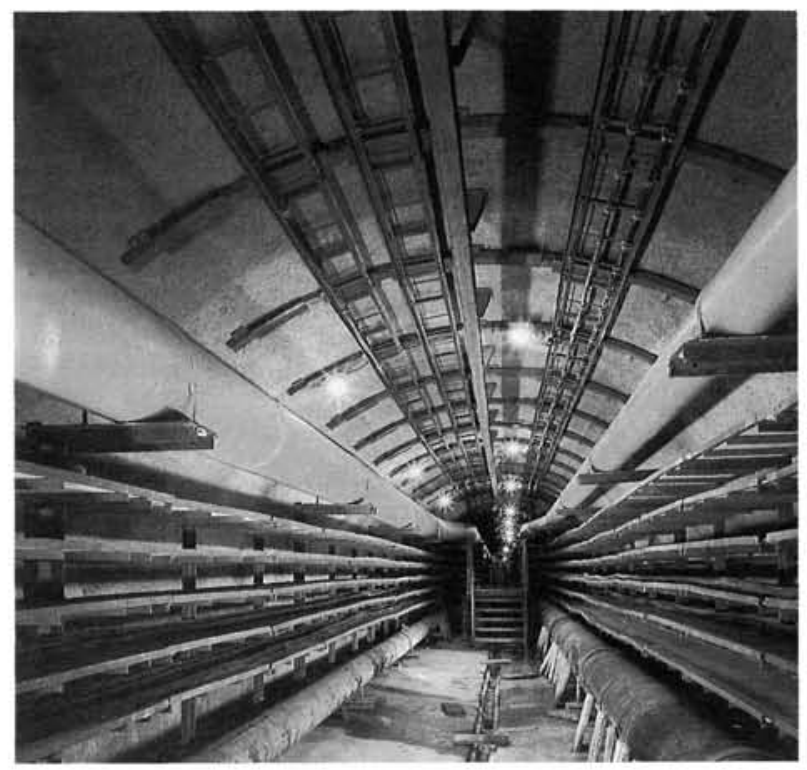

Fig. 8.-Vista de un colector.

http://informesdelaconstruccion.revistas.csic.es 


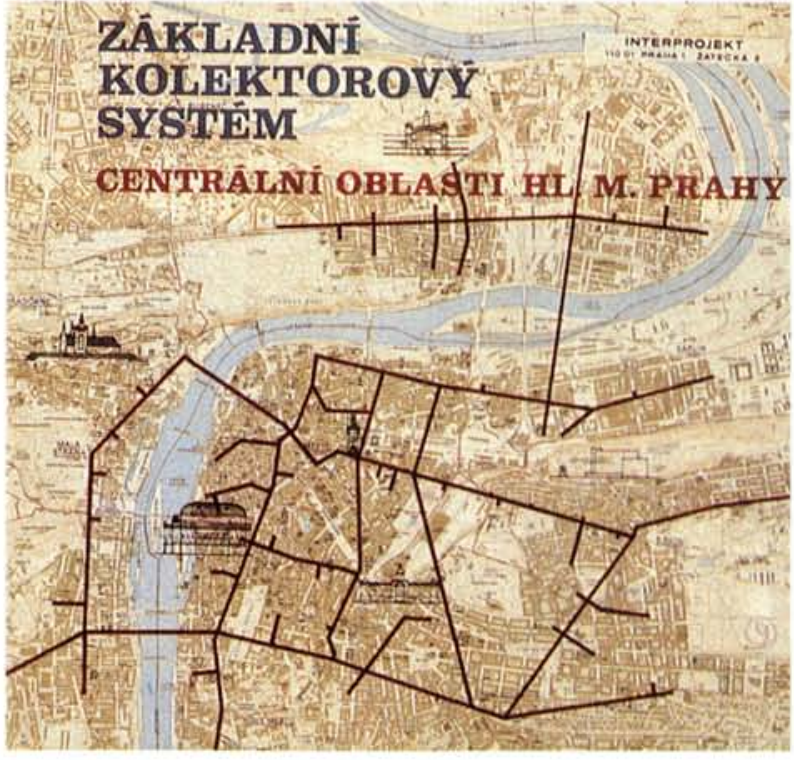

Fig. 9.-Plano del sistema de colectores de Praga.

Otro ejemplo de regeneración de centros históricos realizada en Checoslovaquia es el de Tábor. Esta ciudad medieval, célebre por el importante papel que desempeñó durante el movimiento reformador de los Hussitas, posee un núcleo histórico que fue declarado monumento cultural nacional como primera ciudad checa.

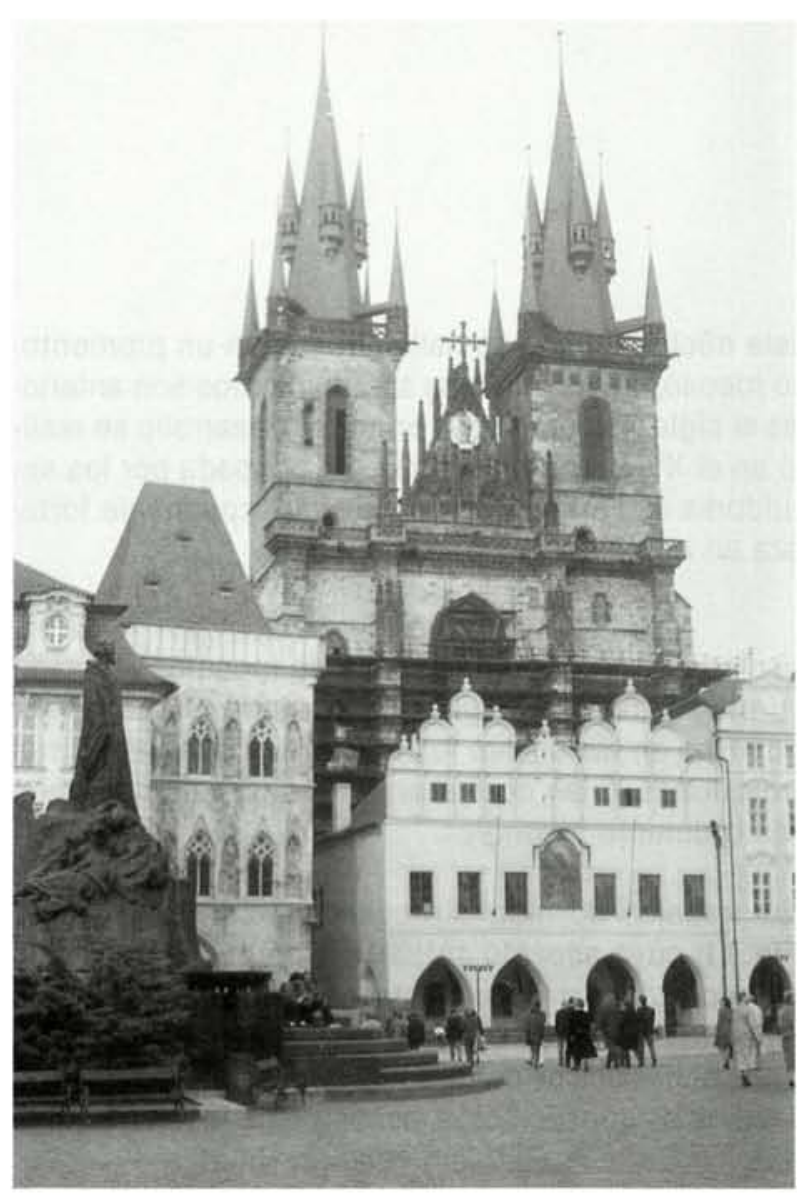

Fig. 10.- La iglesia de Tyn, situada en el centro histórico de Praga.

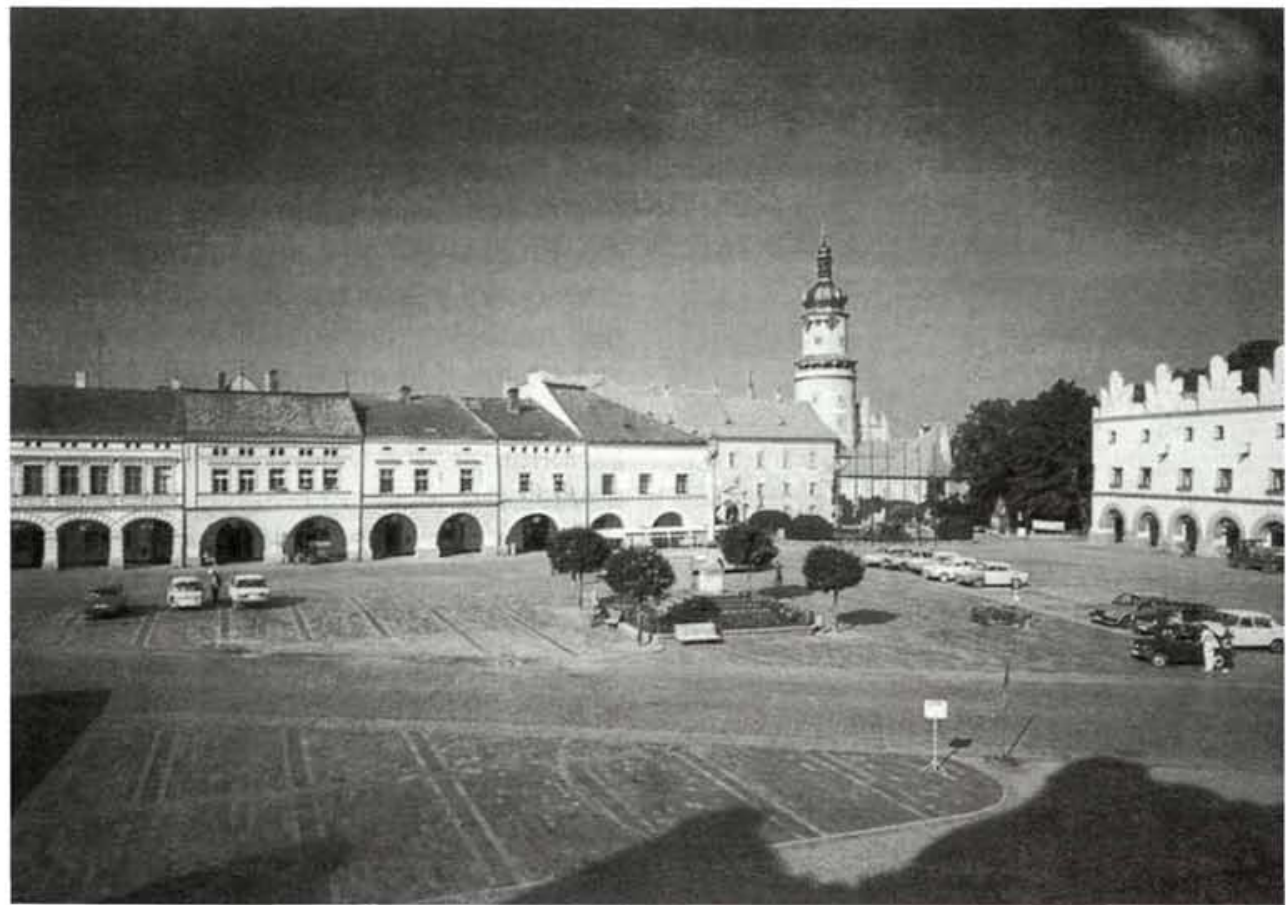

Fig. 11.-Plaza en el centro histórico de Tábor.

(C) Consejo Superior de Investigaciones Científicas 
Este núcleo urbano se halla situado en un promontorio rocoso, cuyos primeros asentamientos son anteriores al siglo XIII, pero cuyo principal desarrollo se realizó en el XV, cuando la ciudad fue ocupada por los seguidores de Huss y convertida en inexpugnable fortaleza en muy poco tiempo.

La ciudad fue construida según un ingenioso trazado y protegida por una amplia red de galerías subterráneas y cuevas en las cuales los habitantes guardaban sus provisiones, o las ocultaban en los turbulentos tiem. pos o durante incendios.

Los trabajos de reconstrucción del centro de Tábor (Fig. 11) cuyo aspecto actual, sin embargo, es de los períodos gótico y renacentista, comenzaron en los años sesenta. El deficiente estado de la mayoria de los edificios históricos se debía a una desidia rutinaria de los trabajos de conservación, asi como de la red de servicios.

El organismo estatal Interprojekt prepara proyectos de reconstrucción de los principales edificios, murallas y baluartes, asi como estudios de consolidación o protección de los espacios subterráneos. Estos espacios, que comprenden las cuevas o bodegas de los edificios, y los corredores de intercomunicación, están dispues-

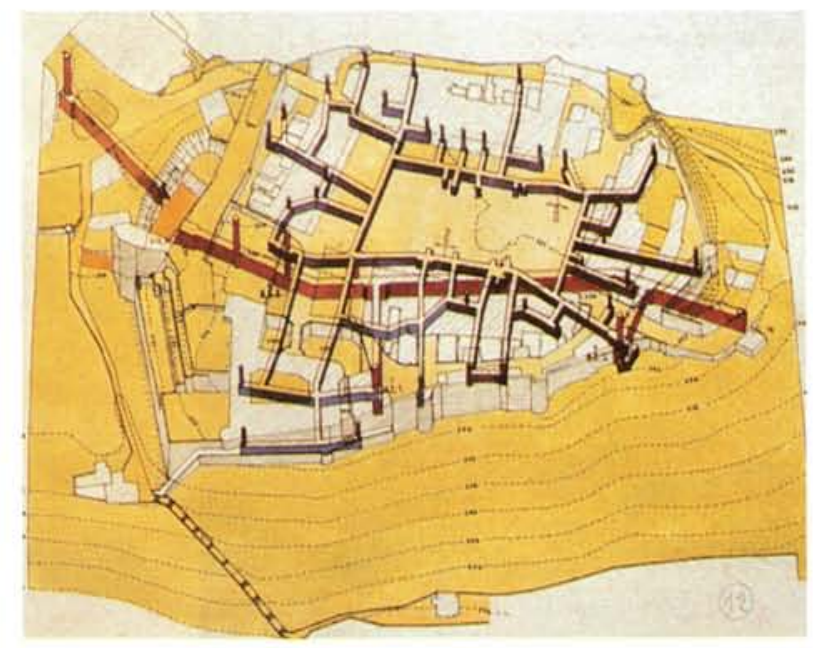

Fig. 12.-Red de colectores de la ciudad de Tábor. (C) Consejo Superior de Investigaciones Científicas Licencia Creative Commons 3.0 España (by-nc)

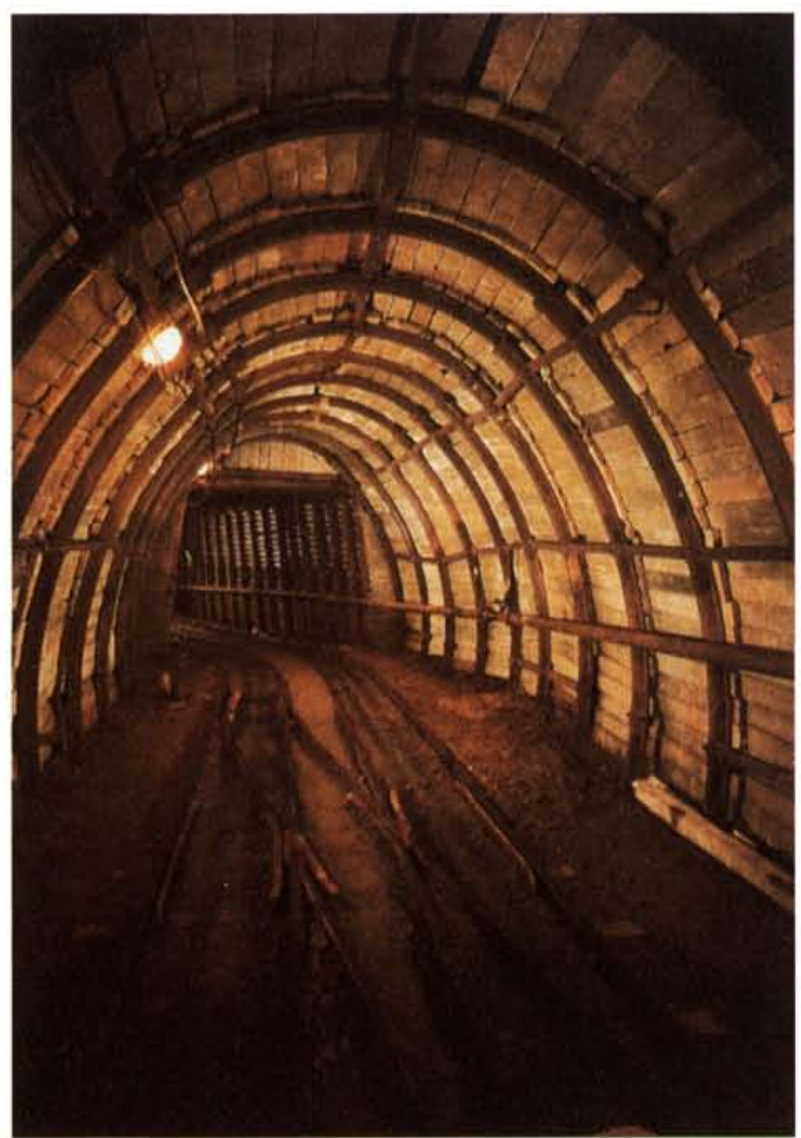

Fig. 13.-Vista de un colector.

tos en Tábor, generalmente, en dos niveles: Las cuevas del superior, de altura comprendida entre 2,6 y 3,0 metros, tienen por techo una bóveda de piedra, y este te. cho constituye el suelo de los edificios situados encima. En el segundo e inferior nivel, están situados los corredores y galerías, dispuestos horizontalmente según múltiples ramificaciones, que entrelazan las cuevas de los diferentes edificios.

La longitud total de las galerías, que han sido hechas accesibles y documentadas geológicamente, es de unos 12 kilómetros, habiéndose preservado galerias originales de una altura media de 1,90 metros y una anchura de 2 metros.

Cuando se termine este vasto proyecto, según los datos aportados en la ponencia de Jana Krausová, el sis. tema de colectores instalado en el lugar de esta red medieval dará servicio a 366 edificios que abarcan una extensión de 28 hectáreas, mediante conductos para agua, saneamiento, energía, iluminación, teléfono y ra. dio (Figs. 12 y 13).

http://informesdelaconstruccion.revistas.csic.es 


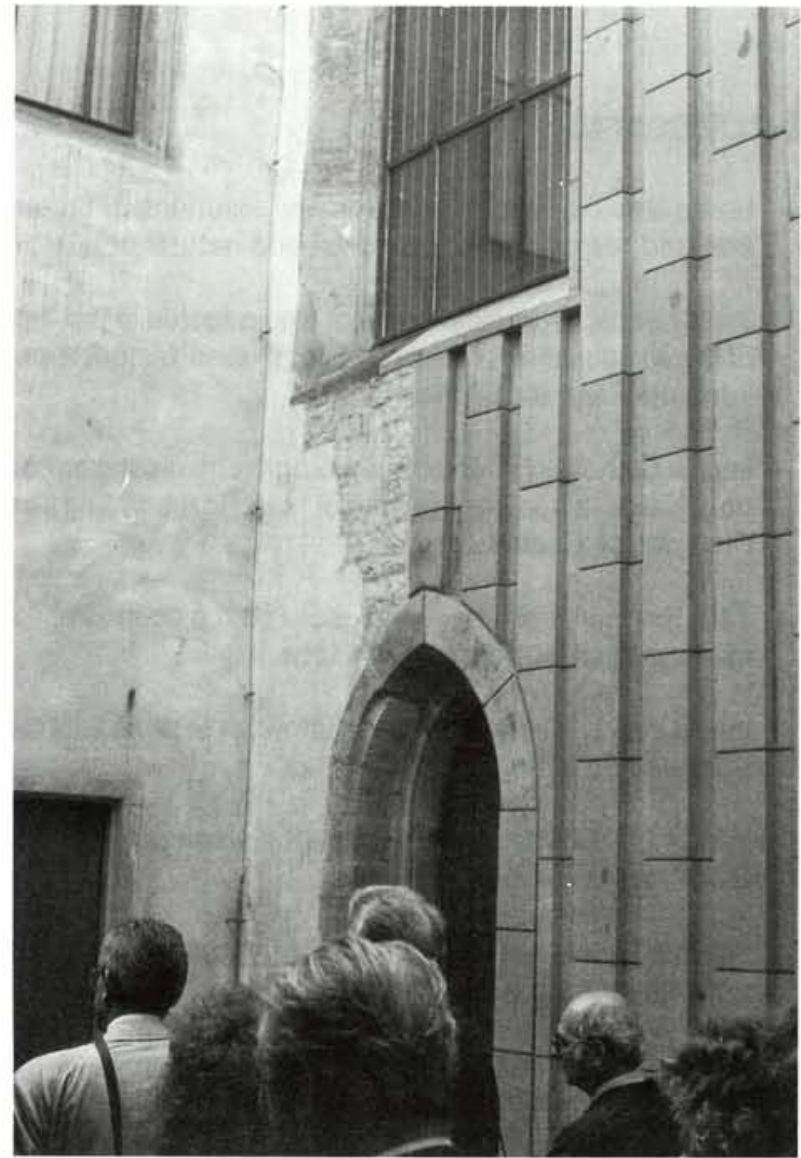

Fig. 14.-Grupo de asistentes al Simposio, viendo la fachada restau rada del medieval convento de Santa Inés (orden del Cister).

En conclusión, puede asegurarse que los resultados de los trabajos geológicos y de ingeniería han permitido determinar las prioridades de preservación de edificios en el núcleo histórico de la ciudad. Y como contribución al éxito de las tareas de rehabilitación, es destacable el cambio cualitativo experimental en las condiciones técnicas de la mayoría de los edificios a partir de 1968 , lo cual se ha reflejado también en el medio ambiente y en la ecología de la histórica ciudad, actualmente foco de atención de los turistas.

Como información final para la presente síntesis de las ponencias presentadas al Simposio de Praga, el ingeniero Ladislav Hapl, CSc y el ingeniero-arquitecto V. Kesnerová, presentaron un trabajo sobre la investigación como primera fase del proceso de regeneración y establecimiento de un sistema de información en edificación y en ingeniería.

(c) Consejo Superior de Investigaciones Científicas Licencia Creative Commons 3.0 España (by-nc)

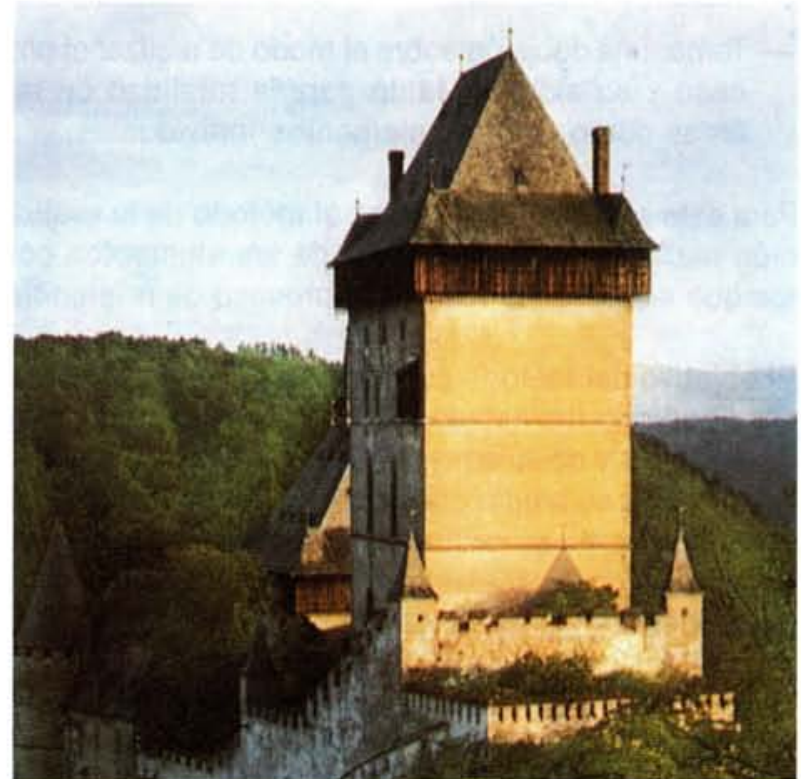

Fig. 15.-La gran torre del Castillo de Karlstejn.

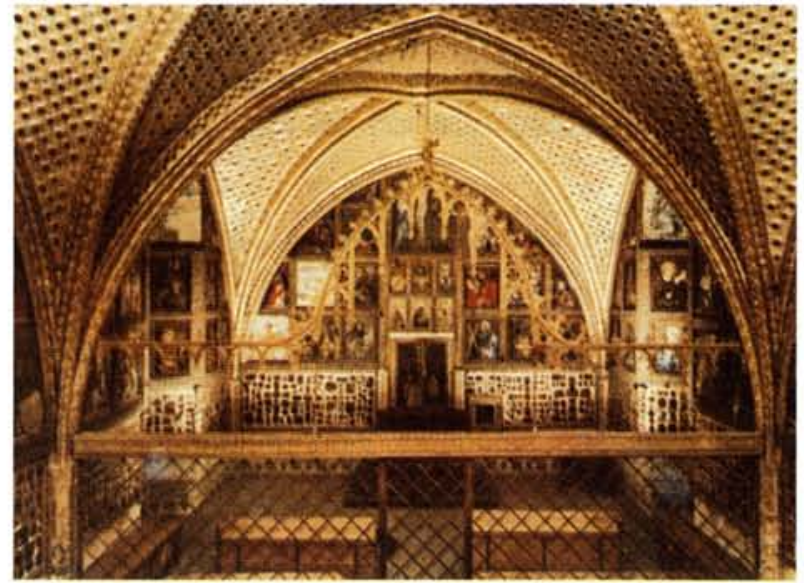

Fig. 16. - La Capilla de Santa Cruz, restaurada, en el interior del Cas. tillo de Karlstein.

En conexión con la preparación de la propuesta "Principios de un sistema de información para el proceso de regeneración de las capacidades existentes de los edificios" se han establecido las exigencias fundamen. tales siguientes:

- Introducir una coordinación de las actividades realizadas por los principales participantes en el proceso de regeneración y de su mutua colaboración. http://informesdelaconstruccion.revistas.csic.es 
- Establecer un pronóstico a largo plazo del desarrollo de solar o del terreno como inicial requisito para la preparación del proceso de regeneración.

- Tratar de hallar una clara apreciación de las cuali. dades existentes del territorio y de sus elementos individuales.

- Elaborar un proyecto alternativo del modo de realizar el proceso de regeneración, considerando todas las influencias y circunstancias incidentes.

- Tomar una decisión sobre el modo de realizar el proceso y su alcance, tanto para la totalidad de las áreas como para los elementos individuales.

Para este programa se emplea el método de la evaluación matemática y estadística de los elementos con los que se pretende realizar el proceso de referencia.

El objetivo del método consiste en presentar un estudio fidedigno del estado material de la capacidad de los edificios y de su conexión con el diseño urbano del territorio, de su arquitectura y de su infraestructura, sin tener en cuenta su probable utilización. El verdadero objetivo es conocer el estado real existente de las capacidades de los edificios examinados.

El método de evaluación matemática y estadistica asis. tido por ordenador (expresión porcentual de los resultados de la compleja apreciación de estado material de los edificios) se emplea en la aplicación del estado material de los elementos.

En resumen: Agradecemos desde estas líneas a la EUROBUILD, la organización del Simposio en Praga, que estimamos supone una contribución útil y sumamente interesante para el seguimiento del tema: "Restauración y rehabilitación de monumentos y ciudades históricos".

\section{BIBLIOGRAFIA}

- Unido-Geoindustria: "Problems and solutions in protec. tion and restoration of historical and natural objets".

- Jan Svejcha: "Technical works of Geoindustria in the field of the safeguarding of important complexes, historical monumentes and rock formations".

- Ludvík Cichovský: "Modern petrologic-chemical diagnostics in redevelopment projects of historical buildings carried out by Geoindustria".

- Jana Krausová: "Engineering geological problems of the medieval part of the town of Tábor.

- Interprojekk: "Conurbations solutions of technical infras. tructures collectors in Prague".

- Interprojekk: "Regeneration of historic centres of towns . Tábor".

- EUROBUILD Proceedings: "Rehabilitation of historical town centres and monuments".

- Ing. Frantisek Dvoraak: "Collectors in the central part of the city of Prague".

- Ing. Ladislav Hapl, CSc.; Ing. arch. Vera Kesnerová: "Building and engineering investigation as the first phase of regenerating process and establishment of information system". 